Pacific Journal of Mathematics

BIJECTIVE PROOFS OF CERTAIN VECTOR PARTITION 


\title{
BIJECTIVE PROOFS OF CERTAIN VECTOR PARTITION IDENTITIES
}

\author{
BRUCE E. SAGAN
}

\begin{abstract}
Known generating functions for certain families of $r$-partite (vector) partitions are derived using a simple combinatorial bijection. This same technique is seen to apply to various new partition identities as well.
\end{abstract}

1. Introduction. Let the nonnegative integers be denoted by $N=\{0,1,2, \cdots\}$. Given $(m, n) \in N^{2}$, a bipartite partition of $(m, n)$ is a component-wise sum

$$
(m, n)=\left(m_{1}, n_{1}\right)+\left(m_{2}, n_{2}\right)+\cdots+\left(m_{k}, n_{k}\right)
$$

where for all $i:\left(m_{i}, n_{i}\right) \in N^{2}-\{(0,0)\}$ and $\left(m_{i}, n_{i}\right) \geqq\left(m_{i+1}, n_{i+1}\right)$ in the lexicographic order on $N^{2}$. The partition (1.1) is restricted if in addition

$$
\min \left(m_{i}, n_{i}\right) \geqq \max \left(m_{i+1}, n_{i+1}\right)
$$

for $1 \leqq i<k$.

L. Carlitz [2] first derived the generating function for restricted bipartite partitions. Subsequently Carlitz and Roselle [3] enumerated certain special families of these partitions e.g., restricted bipartite partitions where the $m_{i}$ and $n_{i}$ are all odd. Finally both Roselle [4] and Andrews [1] have obtained different generalizations for multipartite partitions.

All these results have been proved by manipulation of formal power series. However, the simplicity of the generating functions obtained suggests that purely combinatorial methods could be applied. The purpose of this paper is to give direct bijective proofs which, in addition, permit us to count a new family of partitions.

2. The basic bijection. Let $\pi(m, n)$ be the number of restricted bipartite partitions of $(m, n)$. Then Carlitz's result can be stated as follows:

THEOREM 1 [2]. The generating function for restricted bipartite partitions is

$$
\sum_{m, n \geqq 0} \pi(m, n) X^{m} Y^{n}=\prod_{i>0} \frac{1}{\left(1-X^{i} Y^{i-1}\right)\left(1-X^{i-1} Y^{i}\right)\left(1-X^{2 i} Y^{2 i}\right)} .
$$

Proof. It suffices to find a bijection between restricted bipartite 
partitions of $(m, n)$ and bipartite partitions of $(m, n)$ using vectors from the set $\{(i, i-1) ;(i-1, i) ;(2 i, 2 i) \mid i \in P=N-\{0\}\}$.

Given a restricted bipartite partition of the form (1.1) then

$$
\begin{aligned}
& m=m_{1}+m_{2}+\cdots+m_{k} \\
& n=n_{1}+n_{2}+\cdots+n_{k} .
\end{aligned}
$$

Note that (1.2) guarantees that $m_{i} \geqq m_{i+1}$ and $n_{i} \geqq n_{i+1}$ for $i=$ $1,2, \cdots, k-1$ so that these partitions of $m$ and $n$ are in the canonical (decreasing) order.

Now conjugate (2.1) so that

$$
\begin{aligned}
& m=m_{1}^{\prime}+m_{2}^{\prime}+\cdots \\
& n=n_{1}^{\prime}+n_{2}^{\prime}+\cdots
\end{aligned}
$$

$\left(m_{j}^{\prime}=\left|\left\{i \mid m_{i} \geqq j\right\}\right|\right.$, similarly for $\left.n_{j}^{\prime}\right)$. Hence we can write

$$
(m, n)=\left(m_{1}^{\prime}, n_{1}^{\prime}\right)+\left(m_{2}^{\prime}, n_{2}^{\prime}\right)+\cdots .
$$

We claim that $\left|m_{j}^{\prime}-n_{j}^{\prime}\right| \leqq 1$. For if $m_{j}^{\prime}=i$ then $m_{i} \geqq j$ and $m_{i+1}<j$ (by convention $m_{k+1}=0$ ). From (1.2) we see that $n_{i+2} \leqq$ $m_{i+1}<j$ so $n_{j}^{\prime}<i+2$ and $n_{j}^{\prime}-m_{j}^{\prime} \leqq 1$. Similarly $m_{j}^{\prime}-n_{j}^{\prime} \leqq 1$.

It follows that the partition (2.2) uses vectors from the set $\{(i, i-1) ;(i-1, i) ;(i, i) \mid i \in \boldsymbol{P}\}$. But we can eliminate those vectors of the form $(2 i-1,2 i-1)$ by replacing them with the sum $(i, i-1)+(i-1, i)$. This turns (2.2) into a bipartite partition using the required set of vectors.

To show that the above procedure is bijective, we construct its inverse. Starting with a bipartite partition of $(m, n)$ using vectors from the set $\{(i, i-1) ;(i-1, i) ;(2 i, 2 i) \mid i \in P\}$, we first add together all pairs of vectors of the form $(i, i-1)$ and $(i-1$, i). After putting the resulting partition in lexicographic order, conjugate each component to obtain a new bipartite partition of $(m, n)$. Verifying that this partition is restricted and that this algorithm is indeed the inverse to the preceeding construction is straightforward and left to the reader.

3. The identities of Carlitz and Roselle. It is now easy to derive the generating functions for various special types of restricted bipartite partitions either by modifying the bijection in $\S 2$ or by standard combinatorial tricks.

The vector $\left(m_{i}, n_{i}\right)$ in the bipartite partition (1.1) is called the $i$ th part of the partition and the whole partition is said to have $k$ parts i.e., $k$ nonzero vectors. (Do not confuse this with bipartite which means that each vector has two components.) 
Proposition 2 [3]. The generating function for restricted bipartite partitions with at most $k$ parts is

$$
\xi^{(k)}(X, Y)=\prod_{i=1}^{k} \frac{1-X^{2 i-1} Y^{2 i-1}}{\left(1-X^{i} Y^{i-1}\right)\left(1-X^{i-1} Y^{i}\right)\left(1-X^{i} Y^{i}\right)} .
$$

Proof. Rewrite this identity as

$$
\frac{\xi^{(k)}(X, Y)}{\prod_{i=1}^{k}\left(1-X^{2 i-1} Y^{2 i-1}\right)}=\prod_{i=1}^{k} \frac{1}{\left(1-X^{i} Y^{i-1}\right)\left(1-X^{i-1} Y^{i}\right)\left(1-X^{i} Y^{i}\right)} .
$$

Thus it suffices to find a bijection between pairs of bipartite partitions $\left(\sum_{j}\left(m_{j}, n_{j}\right) ; \sum_{j}\left(p_{j}, q_{j}\right)\right)$ and bipartite partitions $\sum_{j}\left(r_{j}, s_{j}\right)$ where

$\sum_{j}\left(m_{j}, n_{j}\right)$ is restricted and has at most $k$ parts

$\sum_{j}\left(p_{j}, q_{j}\right)$ has parts from the set $\{(2 i-1,2 i-1) \mid 1 \leqq i \leqq k\}$

$\sum_{j}\left(r_{j}, s_{j}\right)$ has parts from $\{(i, i-1) ;(i-1, i) ;(i, i) \mid 1 \leqq i \leqq k\}$

and

$$
\sum_{j}\left(m_{j}+p_{j}\right)=\sum_{j} r_{j}, \sum_{j}\left(n_{j}+q_{j}\right)=\sum_{j} s_{j} .
$$

Using the notation of $\S 2$ and writing $\sum_{j}\left(p_{j}, q_{j}\right)=\sum_{l}\left(2 i_{l}-1\right.$, $\left.2 i_{l}-1\right)$ we can express this bijection as

$$
\begin{aligned}
\left(\sum_{j}\left(m_{j}, n_{j}\right) ; \sum_{l}\left(2 i_{l}-1,2 i_{l}-1\right)\right) & \longleftrightarrow \\
& \sum_{j}\left(m_{j}^{\prime}, n_{j}^{\prime}\right)+\sum_{l}\left(i_{l}, i_{l}-1\right)+\left(i_{l}-1, i_{l}\right) .
\end{aligned}
$$

Note that since $\sum_{j}\left(m_{j}, n_{j}\right)$ has at most $k$ parts we have $m_{j}^{\prime}, n_{j}^{\prime} \leqq k$ and also $i_{l} \leqq k$ for all $l$.

Proposition 3 [3]. The generating function for restricted bipartite partitions where all components are odd is

$$
\sum_{k=0}^{\infty} X^{k} Y^{k} \xi^{(k)}\left(X^{2}, Y^{2}\right)
$$

Proof. Subtracting one from each component of a restricted bipartite partition with exactly $k$ parts and odd components yields a restricted bipartite partition with at most $k$ parts and even components plus a part $(k, k)$. This is a bijection and the proposition follows.

Proposition 4 [2]. The generating function for restricted 
bipartite partitions with distinct parts (i.e., $\min \left(m_{i}, n_{i}\right)>\max$ $\left(m_{i+1}, n_{i+1}\right)$ for all $\left.i\right)$ is

$$
\sum_{k=0}^{\infty}(X Y)^{\left(\begin{array}{l}
k \\
2
\end{array}\right)}\left(1-X^{k} Y^{k}\right) \xi^{(k)}(X, Y) .
$$

Proof. By subtracting $k-i$ from the $i$ th part of a restricted bipartite partition with exactly $k$ distinct parts we obtain a restricted bipartite partition with exactly $k$ parts plus a part of the form $(k-1+k-2+\cdots+1, k-1+k-2+\cdots+1)$. Hence the generating function in question is

$$
\sum_{k=0}^{\infty}(X Y)^{\left({ }^{k}\right)}\left[\xi^{(k)}(X, Y)-\xi^{(k-1)}(X, Y)\right]
$$

where $\xi^{(-1)}(X, Y)=0$.

The same sort of reasoning as in the previous proposition also produces

PROPOSITION 5 [3]. The generating function for restricted bipartite partitions weighted by number of parts is

$$
1+(1-\lambda) \sum_{k=1}^{\infty} \lambda^{k} \xi^{(k)}(X, Y)
$$

i.e., the coefficient of $\lambda^{k} X^{m} Y^{n}$ in the above expansion is the number of restricted bipartite partitions of $(m, n)$ with exactly $k$ parts.

4. Andrews' generalization. Let $r$ be a fixed positive integer and consider $n=\left(n_{1}, n_{2}, \cdots, n_{r}\right) \in N^{r}$. An r-partite or multipartite partition of $n$ is a sum

$$
\boldsymbol{n}=\boldsymbol{n}_{1}+\boldsymbol{n}_{2}+\cdots+\boldsymbol{n}_{k}
$$

where $\boldsymbol{n}_{i} \in \boldsymbol{N}^{r}-\{0\}$ and $\boldsymbol{n}_{i} \geqq \boldsymbol{n}_{i+1}(\mathbf{0}=(0,0, \cdots, 0))$. As in Andrews [1] we define the summatory maximum of $\boldsymbol{m}=\left(m_{1}, m_{2}, \cdots, m_{r}\right)$ by

$$
\operatorname{smax} \boldsymbol{m}=\sum_{j=1}^{r} m_{j}-(\boldsymbol{r}-1) \cdot \min \boldsymbol{m} .
$$

Note that if $r=1$ or 2 then $\operatorname{smax} m=\max m$. Finally the multipartite partition (4.1) is smax restricted if for all $i: \min n_{i} \geqq$ $\operatorname{smax} \boldsymbol{n}_{i+1}$.

In what follows we use the notation $X^{m}=X_{1}^{m_{1}} X_{2}^{m_{2}} \cdots X_{r}^{m_{r}}$ where $\boldsymbol{m}=\left(m_{1}, m_{2}, \cdots, m_{r}\right)$.

THEOREM 6 [1]. If $\pi(n)$ is the number of smax restricted multi- 
partite partitions of $n \in N^{r}$ then

$$
\sum_{n \geq 0} \pi(n) X^{n}=\prod_{i \in I} \frac{1}{1-X^{i}}
$$

where $I=\{(i, i, \cdots, i) \mid i \in \boldsymbol{P}, i \neq 1(\bmod r)\} \cup\{(i+1, i, i, \cdots, i) ;(i, i+1$, $i, \cdots, i) ; \cdots ;(i, i, i, \cdots, i+1) \mid i \in N\}$.

Proof. Proceeding as in the proof of Theorem 1, consider an smax restricted multipartite partition of the form (4.1) where $n_{i}=$ $\left(n_{i 1}, n_{i 2}, \cdots, n_{i r}\right)$. We have $n_{j}=\sum_{i=1}^{k} n_{i j}$ for $1 \leqq j \leqq r$ and $n_{i j} \geqq$ $\min \boldsymbol{n}_{i} \geqq \operatorname{smax} \boldsymbol{n}_{i+1} \geqq \max \boldsymbol{n}_{i+1} \geqq n_{i+1 j}$. Hence if $n_{j}=\sum_{i=1}^{l} n_{i j}^{\prime}$ are the conjugate partitions, one for each component, then

$$
\boldsymbol{n}=\boldsymbol{n}_{1}^{\prime}+\boldsymbol{n}_{2}+\cdots+\boldsymbol{n}_{l}^{\prime}
$$

where $\boldsymbol{n}_{i}^{\prime}=\left(n_{i 1}^{\prime}, n_{i 2}^{\prime}, \cdots, n_{i r}^{\prime}\right)$.

Since $\min \boldsymbol{n}_{i} \geqq \max \boldsymbol{n}_{i+1}$ for all $1 \leqq i<k$ it follows that $\mid n_{i s}^{\prime}-$ $n_{i t}^{\prime} \mid \leqq 1$ for all $1 \leqq s, t \leqq r$. It is convenient to have a notation for the vectors appearing in (4.2): given $T \varsubsetneqq\{1,2, \cdots, r\}$ and $i \in N$ let

$$
\boldsymbol{m}_{T}(i)=\left(m_{1}, m_{2}, \cdots, m_{r}\right) \text { where } m_{j}=\left\{\begin{array}{lll}
i & \text { if } & j \notin T \\
i+1 & \text { if } & j \in T .
\end{array}\right.
$$

If $T=\{t\}$ we write $\boldsymbol{m}_{t}(i)$ for $\boldsymbol{m}_{\{t\}}(i)$ and if $T=\phi$ we shorten $\boldsymbol{m}_{\dot{p}}(i)$ to $m(i)$.

Now given $m_{T}(i)$ in (4.2) with $T \neq \phi$ we claim that (4.2) also contains at least $|T|-1$ other parts of the form $m(i)$. To see that this is the case, we compute

$$
\begin{aligned}
|\{\boldsymbol{m}(i)\}| & =\min \boldsymbol{n}_{i}-\max \boldsymbol{n}_{i+1} \\
& \geqq \operatorname{smax} \boldsymbol{n}_{i+1}-\max \boldsymbol{n}_{i+1} \\
& =\left[\sum_{j=1}^{r}\left(n_{i+1 j}-\min \boldsymbol{n}_{i+1}\right)\right]-\left(\max \boldsymbol{n}_{i+1}-\min \boldsymbol{n}_{i+1}\right) \\
& =\sum(|T|-1)
\end{aligned}
$$

where the last sum is over all $m_{T}(i)$ in (4.2) with $T \neq \phi$. Hence we may replace $\boldsymbol{m}_{T}(i)+\boldsymbol{m}(i)+\boldsymbol{m}(i)+\cdots+\boldsymbol{m}(i)=\boldsymbol{m}_{T}(i)+(|\boldsymbol{T}|-1) \cdot \boldsymbol{m}(i)$ by the sum $\sum_{t \in T} \boldsymbol{m}_{t}(i)$ where, by definition, $\boldsymbol{m}_{t}(i) \in I$.

After all the parts $\boldsymbol{m}_{T}(i), T \neq \phi$, have been replaced, we consider any remaining parts of the form $\boldsymbol{m}(i)$ with $i=q r+1$. Using $\boldsymbol{m}(i)=\sum_{t=1}^{r} \boldsymbol{m}_{t}(q)$ we can complete the transformation of (4.2) into a multipartite partition using only the parts in $I$.

To reverse the above construction, we start with a multipartite partition

$$
\boldsymbol{n}=\boldsymbol{m}_{1}+\boldsymbol{m}_{2}+\cdots+\boldsymbol{m}_{k}
$$


where $\boldsymbol{m}_{i} \in I$ for all $i$. Now consider the sets $T_{1 i}=\left\{t \mid \boldsymbol{m}_{t}(i)\right.$ is a part of (4.3)\} and replace $\sum_{t \in T_{1 i}} m_{t}(i)$ by $m_{T_{1 i}}(i)$ if $\phi \varsubsetneqq T_{1 i} \varsubsetneqq\{1,2, \cdots, r\}$ or by $\boldsymbol{m}(\boldsymbol{r} i+1)$ if $T_{1 i}=\{1,2, \cdots, r\}$ (if $T_{1 i}=\phi$ there is nothing to do). Now we have a new sum

$$
\boldsymbol{n}=\boldsymbol{m}_{1}^{(1)}+\boldsymbol{m}_{2}^{(1)}+\cdots+\boldsymbol{m}_{l}^{(1)}
$$

so let $T_{2 i}=\left\{t \mid m_{t}(i)\right.$ is a part of (4.4)\} and apply the same replacement rules to $\sum_{t \in T_{2 i}} m_{t}(i)$.

After a finite number of iterations we will have

$$
\boldsymbol{n}=\boldsymbol{m}_{1}^{(s)}+\boldsymbol{m}_{2}^{(s)}+\cdots+\boldsymbol{m}_{p}^{(s)}
$$

with $\left|T_{s+1 i}\right| \leqq 1$ for all $i$. Arranging (4.5) in lexicographic order, we may then conjugate each component separately to obtain a multipartite partition of $n$ which will be restricted because $T_{1 i} \supseteqq$ $T_{2 i} \supseteqq \cdots \supseteqq T_{s i}$.

5. New generating functions. The following proposition generalizes the identities of Carlitz and Roselle ( $(3)$ in the same way that Andrews' Theorem (number 6 above) generalizes that of Carlitz (Theorem 1). The same methods of proof can be applied so we merely state the results.

Proposition $7 \mathrm{~A}$. The generating function for smax restricted $r$-partite partitions with at most $k$ parts is

$$
\xi^{(k)}\left(X_{1}, \cdots, X_{r}\right)=\prod_{i=0}^{k-1} \frac{1-X^{m(r i+1)}}{\left(1-X^{m(i+1)}\right) \prod_{t=1}^{r}\left(1-X^{m_{t}(i)}\right)} .
$$

B. The generating function for smax restricted $r$-partite partitions with all components odd is

$$
\sum_{k=0}^{\infty} X^{m(k)} \xi^{(k)}\left(X_{1}^{2}, \cdots, X_{r}^{2}\right) .
$$

C. The generating function for smax restricted $r$-partite partitions with distinct parts $\left(\min \boldsymbol{n}_{i}>\operatorname{smax} \boldsymbol{n}_{i+1}\right)$ is

$$
\sum_{k=0}^{\infty} X^{m\left(\left(\begin{array}{c}
k \\
2
\end{array}\right)\right)}\left(1-X^{m(k)}\right) \xi^{(k)}\left(X_{1}, \cdots, X_{r}\right) .
$$

D. The generating function for smax restricted $r$-partite partitions weighted by the number of parts is

$$
1+(1-\lambda) \sum_{k=1}^{\infty} \lambda^{k} \xi^{(k)}\left(X_{1}, \cdots, X_{r}\right) .
$$


We are now led to define the "dual" notion to the summatory maximum. Given $\boldsymbol{m}=\left(m_{1}, m_{2}, \cdots, m_{r}\right) \in \boldsymbol{N}^{r}$ the summatory minimum of $\boldsymbol{m}$ is defined by

$$
\operatorname{smin} \boldsymbol{m}=\sum_{j=1}^{r} m_{j}-(\boldsymbol{r}-1) \max \boldsymbol{m} .
$$

When $r=1$ or 2 we have $\operatorname{smin} \boldsymbol{m}=\min \boldsymbol{m}$.

An $r$-partite partition (4.1) is smin restricted if $\operatorname{smin} \boldsymbol{n}_{i} \geqq$ $\max \boldsymbol{n}_{i+1}$ for $1 \leqq i \leqq k$ (by convention $\max \boldsymbol{n}_{k+1}=0$ ). The analogue of Theorem 6 is

THEOREM 8 . Let $\pi^{*}(\boldsymbol{n})$ be the number of smin restricted $r$-partite partitions of $\boldsymbol{n}$, then

$$
\sum_{n \geq 0} \pi^{*}(\boldsymbol{n}) X^{n}=\prod_{i \in I^{*}} \frac{1}{1-X^{i}}
$$

where $I^{*}=\{(i, i, i, \cdots, i) \mid i \in \boldsymbol{P}, i \not \equiv-1(\bmod r)\} \cup\{(i-1, i, i, \cdots, i)$; $(i, i-1, i, \cdots, i) ; \cdots ;(i, i, i, \cdots, i-1) \mid i \in \boldsymbol{P}\}$.

Similarly, we can dualize Proposition 7:

Proposition $9 \mathrm{~A}$. The generating function for smin restricted r-partite partitions with at most $k$ parts is

$$
\zeta^{(k)}\left(X_{1}, \cdots, X_{r}\right)=\prod_{i=1}^{k} \frac{1-X^{m(r i-1)}}{\left(1-X^{m(i)}\right) \prod_{t=1}^{r}\left(1-X^{m_{-t^{(i)}}}\right)}
$$

where $\boldsymbol{m}_{-t}(i)=\left(m_{1}, m_{2}, \cdots, m_{r}\right)$ with $m_{i}=\left\{\begin{array}{cl}i-1 & \text { if } j=t \\ i & \text { otherwise. }\end{array}\right.$

B. The generating function for smin restricted $r$-partite partitions with all components odd is

$$
\sum_{k=0}^{\infty} X^{m(k)} \zeta^{(k)}\left(X_{1}^{2}, X_{2}^{2}, \cdots, X_{r}^{2}\right)
$$

C. The generating function for smin restricted $r$-partite partitions with distinct parts is

$$
\left.\sum_{k=0}^{\infty} X^{m}\left(\begin{array}{l}
k \\
2
\end{array}\right)\right)\left(1-X^{m(k)}\right) \zeta^{(k)}\left(X_{1}, \cdots, X_{r}\right) .
$$

D. The generating function for smin restricted r-partite partitions weighted by the number of parts is

$$
1+(1-\lambda) \sum_{k=1}^{\infty} \lambda^{k} \zeta^{(k)}\left(X_{1}, \cdots, X_{r}\right)
$$

Again, the techniques of $\S \S 2$ and 3 can be applied to prove 
the above results. However we should mention the generalization of Roselle [4] which we have been unable to derive using these methods. An $r$-partite partition (4.1) is restricted if $\min \boldsymbol{n}_{i} \geqq$ $\max \boldsymbol{n}_{i+1}$ for $1 \leqq i \leqq k$.

THEOREM 10 [4]. The generating function for restricted $r$-partite pertitions is

$$
\prod_{j=1}^{r} \frac{1}{1-X_{j}} \cdot \prod_{i=1}^{\infty} \frac{1}{X^{m(i)}} H_{r}\left(\frac{1}{X^{m(i)}} ; X_{1}, \cdots, X_{r}\right)
$$

where $H_{r}\left(Y ; X_{1}, \cdots, X_{r}\right)$ is the generalized $q$-Eulerian function defined by the umbral recursion $H_{0}=1$ and $Y \cdot H_{r}=\prod_{j=1}^{r}\left(1+X_{j} H\right)$.

A combinatorial proof in this setting would be very interesting but may be difficult to obtain because the generating function in question has a nontrivial numerator.

\section{REFERENCES}

1. G. E. Andrews, An extension of Carlitz's bipartition identity, Proc. Amer. Math. Soc., 63 (1977), 180-184.

2. L. Carlitz, A problem in partitions, Duke Math. J., 30 (1963), 202-213.

3. L. Carlitz and D. P. Roselle, Restricted bipartite partitions, Pacific J. Math., 19 (1966), 221-228.

4. D. P. Roselle, Restricted k-partite partitions, Math. Nachr., 32 (1966), 139-148.

5. - Generalized Eulerian functions and a problem in partitions, Duke Math. J., 33 (1966), 293-304.

Received July 10, 1981. Research supported in part by NSF Grant MCS 80-03027.

UNIVERSITY OF MICHIGAN

ANN ARBor, MI 48109 


\section{PACIFIC JOURNAL OF MATHEMATICS}

\section{EDITORS}

DONALD BABBITT (Managing Editor)

University of California

Los Angeles, California 90024

HUGO ROSSI

University of Utah

Salt Lake City, UT 84112

C. C. MOORE and ARTHur AGUS

University of California

Berkeley, CA 94720
J. DUGUNDJI

Department of Mathematics

University of Southern California

Los Angeles, California 90007

R. FINN and J. MILGRAM

Stanford University

Stanford, California 94305

\section{ASSOCIATE EDITORS}
R. Arnes
E. F. BECKENBACH
B. H. NeumanN
F. WOLF
K. YösHIDA

\section{SUPPORTING INSTITUTIONS}

UNIVERSITY OF ARIZONA

UNIVERSITY OF BRITISH COLUMBIA

CALIFORNIA INSTITUTE OF TECHNOLOGY

UNIVERSITY OF CALIFORNIA

MONTANA STATE UNIVERSITY

UNIVERSITY OF NEVADA, RENO

NEW MEXICO STATE UNIVERSITY

OREGON STATE UNIVERSITY
UNIVERSITY OF OREGON

UNIVERSITY OF SOUTHERN CALIFORNIA

STANFORD UNIVERSITY

UNIVERSITY OF HAWAII

UNIVERSITY OF TOKYO

UNIVERSITY OF UTAH

WASHINGTON STATE UNIVERSITY

UNIVERSITY OF WASHINGTON 


\section{Pacific Journal of Mathematics}

Vol. 102, No. $1 \quad$ January, 1982

S. Agou, Degré minimum des polynômes $f\left(\sum_{i=0}^{m} a_{i} X^{p^{r i}}\right)$ sur les corps finis

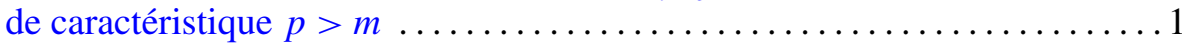

Chi Cheng Chen, On the image of the generalized Gauss map of a complete

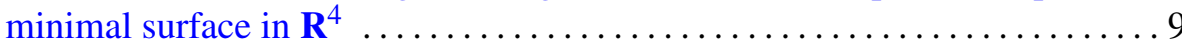

Thomas Curtis Craven and George Leslie Csordas, On the number of real

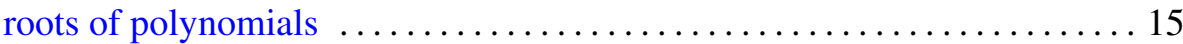

Allan L. Edelson and Kurt Kreith, Nonlinear relationships between oscillation and asymptotic behavior ....................... 29

B. Felzenszwalb and Antonio Giambruno, A commutativity theorem for

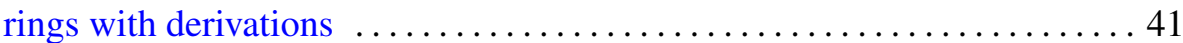

Richard Elam Heisey, Manifolds modelled on the direct limit of lines . . . . . 47

Steve J. Kaplan, Twisting to algebraically slice knots $\ldots \ldots \ldots \ldots \ldots \ldots 5$

Jeffrey C. Lagarias, Best simultaneous Diophantine approximations. II.

Behavior of consecutive best approximations $\ldots \ldots \ldots \ldots \ldots \ldots \ldots 61$

Masahiko Miyamoto, An affirmative answer to Glauberman's conjecture . . . 889

Thomas Bourque Muenzenberger, Raymond Earl Smithson and L. E.

Ward, Characterizations of arboroids and dendritic spaces ........... 107

William Leslie Pardon, The exact sequence of a localization for Witt

groups. II. Numerical invariants of odd-dimensional surgery

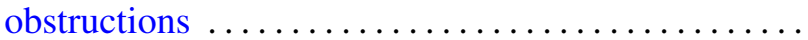

Bruce Eli Sagan, Bijective proofs of certain vector partition identities

Kichi-Suke Saito, Automorphisms and nonselfadjoint crossed products ...

John Joseph Sarraille, Module finiteness of low-dimensional PI rings ...

Gary Roy Spoar, Differentiable curves of cyclic order four . .

William Charles Waterhouse, Automorphisms of quotients of $\Pi \mathrm{GL}\left(n_{i}\right)$

Leslie Wilson, Mapgerms infinitely determined with respect to right-left equivalence

Rahman Mahmoud Younis, Interpolation in strongly logmodular

algebras 